\title{
Possibilidade de Aplicação de Misturas de Herbicidas de AÇÃo Total com Jato Dirigido em Mamoneira de PoRTe Anão ${ }^{1}$
}

\author{
Possibility of Applaying Total Action Herbicide Tank Mixture using Direct Sprayer on Dwarf \\ Internode Castor Bean
}

\author{
MACIEL, C.D.G. ${ }^{2}$, POLETINE, J.P. ${ }^{2}$, VELINI, E.D. ${ }^{3}$, AMARAL, J.G.C. ${ }^{4}$, ZANI, L.P. ${ }^{5}$, SANTOS, R.F. ${ }^{5}$, \\ RODRIGUES, M. ${ }^{6}$, RAIMONDI, M.A. ${ }^{6}$ e RIBEIRO, R.B. ${ }^{\circ}$
}

\begin{abstract}
RESUMO - Com o objetivo de avaliar os efeitos da aplicação em jato dirigido com e sem proteção do bico de pulverização através de chapéu-de-napoleão para misturas de herbicidas de ação total na cultura da mamoneira de porte anão, na safra 2004/2005, um experimento foi conduzido no município de Garça-SP, utilizando-se o híbrido Lyra e espaçamento de 1,0 x 0,5 m. O delineamento experimental utilizado foi o de blocos casualizados, com tratamentos em esquema fatorial $2 \times 10$, com quatro repetições, em que o primeiro fator representou a forma de aplicação de jato dirigido com e sem uso de chapéu-de-napoleão e o segundo os herbicidas: glyphosate $\left(0,72 \mathrm{~kg} \mathrm{ha}^{-1}\right)$; glyphosate $+2,4-\mathrm{D}\left(0,72+0,35 \mathrm{~kg} \mathrm{ha}^{-1}\right)$; glyphosate + flumioxazin $\left(0,72+0,025 \mathrm{~kg} \mathrm{ha}^{-1}\right)$; glyphosate + carfentrazone-ethyl $\left(0,72+0,016 \mathrm{~kg} \mathrm{ha}^{-1}\right)$; glyphosate + diuron $\left(0,72+0,75 \mathrm{~kg} \mathrm{ha}^{-1}\right)$; MSMA + diuron $\left(1,44+0,75 \mathrm{~kg} \mathrm{ha}^{-1}\right)$; paraquat + diuron $\left(0,9 \mathrm{~kg} \mathrm{ha}^{-1}\right)$; paraquat + diquat $\left(0,20+0,20 \mathrm{~kg} \mathrm{ha}^{-1}\right)$; paraquat + bentazon $(0,40+$ $0,48 \mathrm{~kg} \mathrm{ha}^{-1}$ ); e testemunha capinada. A aplicação dos herbicidas foi feita em pós-emergência das plantas daninhas, nas entrelinhas da cultura, utilizando-se pulverizador costal pressurizado com $\mathrm{CO}_{2}$, com uma ponta XR 8002-VS para o uso de chapéu-de-napoleão e duas pontas XR 11002 -VS, espaçadas de $50 \mathrm{~cm}$, para condição sem proteção, ambas com consumo de calda equivalente a $200 \mathrm{~L} \mathrm{ha}^{-1}$. O herbicida glyphosate e as misturas de paraquat + bentazon, glyphosate + 2,4-D e paraquat + diquat proporcionaram os maiores niveis de produtividade da mamoneira Lyra quando aplicados em jato dirigido e com auxilio de chapéude-napoleão. As misturas paraquat + bentazon $\left(0,40+0,48 \mathrm{~kg} \mathrm{ha}^{-1}\right)$ e paraquat + diquat $\left(0,20+0,20 \mathrm{~kg} \mathrm{ha}^{-1}\right)$ demonstraram ser as mais indicadas para aplicação em jato dirigido com proteção do bico de pulverização.
\end{abstract}

Palavras-chave: Ricinus communis, plantas daninhas, seletividade, produtividade.

\begin{abstract}
An experiment was carried out in Garca-SP to evaluate the effects of application using a direct sprayer with and without "Napoleons's hat" protection for total action herbicide tank mixtures on dwarf internode castor bean crop during the 2004/2005 agricultural year, with Lyra hybrid in a $1.0 \times 0.5 \mathrm{~m}$ spacing. The experimental design was randomized complete blocks, with treatments in a factorial scheme $2 \times 10$, with four replications, the first factor being direct sprayer application with and without "Napoleon's hat" protection and the second application of the herbicides glyphosate $\left.(0.72 \mathrm{~kg} \mathrm{ha})^{-1}\right) ;$ glyphosate + 2, 4-D (0.72 + $\left.\left.0.35 \mathrm{~kg} \mathrm{ha}\right)^{1}\right) ;$ glyphosate + flumioxazin $\left(0.72+0.025 \mathrm{~kg} \mathrm{ha}^{-1}\right) ;$ glyphosate + carfentrazone-ethyl $\left(0.72+0.016 \mathrm{~kg} \mathrm{ha}^{-1}\right) ;$ glyphosate + diuron

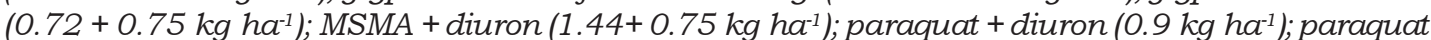
+ diquat $\left(0.20+0.20 \mathrm{~kg} \mathrm{ha} \mathrm{h}^{-1}\right)$; paraquat + bentazon $\left(0.40+0.48 \mathrm{~kg} \mathrm{ha}^{-1}\right)$ and hand weeded check. Herbicide application was accomplished in weed post-emergence, between crop lines, using a carbon dioxide-pressurized backpack sprayer, with one XR 8002-VS nozzle for "Napoleon's
\end{abstract}

1 Recebido para publicação em 21.9.2007 e na forma revisada em 31.3.2008

2 Prof., Dr., Escola Superior de Agronomia de Paraguaçu Paulista - ESAPP, <macielconsultoria@hotmail.com>; ${ }^{3}$ Prof., Dr., Faculdade de Ciências Agronômicas - FCA/Universidade Estadual Paulista, Botucatu-SP, <velini@fca.unesp.br $>$; ${ }^{4}$ Dr., Centro de Testes, Avaliação e Divulgação - CETADI, Coordenadoria de Assistência Técnica Integral, SAA-SP; ${ }^{5}$ Aluno do Curso de Agronomia Faculdade de Agronomia e Engenharia Florestal de Garça - FAEF; ${ }^{6}$ Aluno do Curso de Agronomia - ESAPP, Rua Prefeito Jayme Monteiro, 791, Paraguaçu Paulista, SP.

Planta Daninha, Viçosa-MG, v. 26, n. 2, p. 457-464, 2008 
hat" protection and two XR $11002-V S$ nozzles, $50 \mathrm{~cm}$ spaced, without protection, both using $200 \mathrm{~L}$ ha${ }^{1}$ of water. Glyphosate and paraquat + bentazon, glyphosate +2,4-D and paraquat + diquat tank mixtures provided the highest yield levels for Lyra castor bean hybrid, when applied using a direct sprayer with "Napoleon's hat" protection. Paraquat + bentazon $(0 . .4+0.48 \mathrm{~kg} \mathrm{ha-1)}$ and paraquat + diquat $\left(0.20+0.20 \mathrm{~kg} \mathrm{ha}^{-1}\right)$ tank mixtures were the most indicated for direct sprayer with nozzle protection.

Keywords: Ricinus communis, weed, selectivity, yield.

\section{INTRODUÇÃO}

A mamoneira (Ricinus communis) pertence à família Euphorbiaceae, possivelmente originária da antiga Abissinia, hoje Etiópia. É uma planta de hábito arbustivo, com diversas colorações de caule, folhas e racemos. Seus frutos possuem espinho e não oferecem resistência mecânica. As sementes apresentam diferentes tamanhos, formatos e grande variabilidade de coloração, sendo uma fonte praticamente pura de ácido ricinoléico (Beltrão et al., 2001). Segundo Freire (2001), a ricinoquímica é responsável pela produção de mais de 400 subprodutos, como plásticos, fibras sintéticas, tintas, esmaltes, lubrificantes, além de servir de matéria-prima na produção de próteses em substituição à platina, com custo reduzido, cimento ósseo, ação fungicida e bactericida. Esse ácido também pode ser utilizado como fonte alternativa de combustivel na fabricação do biodiesel, o que torna a cultura importante do ponto de vista econômico e estratégico para o Brasil (Savy Filho, 1998). Além disso, do fruto obtémse a torta de mamona, a qual é utilizada como adubo orgânico (Beltrão et al., 2001).

Em se tratando dos aspectos culturais, mais especificamente com relação ao manejo de plantas daninhas, o cenário nacional ainda é empírico e deficiente, havendo muita perda de rendimento devido à ausência de informações (Azevedo et al., 2001). Segundo Weiss (1983), a mamoneira é considerada uma espécie sensível à competição das plantas daninhas pelos recursos naturais, como umidade do solo, luz, nutrientes e $\mathrm{CO}_{2}$.

O uso de herbicidas na cultura da mamona, apesar de não ser o mais difundido entre os produtores, provavelmente, é o método de controle mais prático e econômico de manejo das plantas daninhas, principalmente para cultivos mais tecnificados de grandes áreas, como é o caso dos híbridos de mamoneira de porte anão. Entre as opções recomendadas encontram-se na maioria produtos utilizados em pré-emergência, como alachlor, diuron, linuron, EPTC, simazine, trifluralin (Weiss, 1983; Gemini, 1985) e 2,4-D isolado ou em mistura com diuron (Yaroslavskaya, 1986). Martins et al. (2004) verificaram que a variedade de mamoneira AL Guarany 2002 foi tolerante aos herbicidas fluazifop-p-butilico, sethoxydim, haloxyfop-methyl, clethodim+fenoxaprop-p-ethyl, quizalofop-p-ethyl, clethodim, propaquizafop, tetraloxydim e butroxydim, utilizados no controle de espécies gramíneas. Maciel et al. (2007) também mencionaram a seletividade de cultivares de mamoneira aos herbicidas pendimethalin, alachlor+pendimethalin e alachlor+trifluralin em aplicações de pré-plantio incorporado e de clomazone e clomazone+trifluralin na pré-emergência da cultura.

No entanto, são inexistentes na literatura estudos sobre o uso de herbicidas de diferentes mecanismos de ação, para controle de plantas daninhas na cultura da mamona, por meio de tecnologias de aplicação em jato dirigido, as quais permitam viabilizar a seletividade de produtos de ação total.

Dessa forma, o trabalho teve como objetivo avaliar os efeitos da aplicação em jato dirigido, com e sem a proteção do bico de pulverização por meio do uso de chapéu-de-napoleão para misturas de herbicidas de ação total, no controle de plantas daninhas da cultura da mamoneira de porte anão.

\section{MATERIAL E MÉTODOS}

O trabalho foi conduzido na área experimental da Fazenda Coração da Terra, localizada no município de Garça-SP, a 663 metros de 
altitude, nas coordenadas $22^{\circ} 12^{\prime} 55^{\prime \prime}$ latitude sul e $49^{\circ} 39^{\prime} 04^{\prime \prime}$ longitude oeste, em solo classificado como Latossolo Vermelho distrófico (LVdf) de textura arenosa.

O cultivar de mamona Lyra foi semeado manualmente em 15/12/2004, utilizando-se três sementes por cova e espaçamento de $1,00 \mathrm{~m}$ entre linhas e $0,50 \mathrm{~m}$ entre plantas. Aos 20 dias após a semeadura foi efetuado o desbaste, deixando uma planta/cova de semeadura. A adubação realizada na semeadura foi com $600 \mathrm{~kg} \mathrm{ha}^{-1}$ da fórmula 04-30-10, e aos 25 dias após a emergência foi efetuada uma adubação de cobertura com uréia na dose de $70 \mathrm{~kg} \mathrm{ha} \mathrm{h}^{-1}$. Os demais tratos culturais foram realizados conforme informações e recomendações técnicas para cultura da mamona (Amaral, 2002).

O delineamento experimental utilizado foi o de blocos casualizados, com quatro repetições, em esquema fatorial $2 \times 10$, sendo duas as formas de aplicação em jato dirigido com e sem o uso de chapéu-de-napoleão e dez as misturas de herbicidas, descritas na Tabela 1. As unidades experimentais constituíram-se de parcelas $\left(24,0 \mathrm{~m}^{2}\right)$ e subparcelas de três linhas da cultura $\left(12,0 \mathrm{~m}^{2}\right)$; as subparcelas foram separadas lateralmente por uma linha da cultura, dividindo as duas condições de aplicação em jato dirigido. As linhas laterais das parcelas foram consideradas como bordaduras, e as duas linhas centrais das subparcelas, como área útil para avaliações.

A aplicação dos herbicidas foi realizada em 24/1/2005, na pós-emergência das plantas daninhas localizadas somente nas entrelinhas da cultura, com as pontas de pulverização posicionadas a $15 \mathrm{~cm}$ de altura do solo, para os sistemas com e sem proteção do chapéu-denapoleão. Para aplicação, utilizou-se um pulverizador costal pressurizado à base de $\mathrm{CO}_{2}$, equipado com barra de uma ponta XR 8002VS para condição com proteção do bico de pulverização por chapéu-de-napoleão e duas pontas XR 11002-VS, espaçadas de $50 \mathrm{~cm}$, para condição sem proteção. As pulverizações foram operadas com consumo de calda equivalente a $200 \mathrm{~L} \mathrm{ha}^{-1}$. Para padronização das condições de pulverização em jato dirigido, com o uso do chapéu-de-napoleão foram efetuadas aplicações em ambos os lados das entrelinhas da mamoneira, para cobertura completa da região representada pelo espaçamento de $1,0 \mathrm{~m}$, de forma semelhante à desenvolvida com a barra de dois bicos na ausência da proteção do chapéu-de-napoleão, em que a cobertura foi efetuada com apenas uma aplicação. No mesmo sentido, a testemunha capinada foi mantida no limpo, a partir do dia da aplicação dos tratamentos químicos, e as comparações de eficácia de controle da infestação foram realizadas utilizando-se como parâmetro de análise a linha divisória das subparcelas, as quais foram mantidas com infestação durante todo o desenvolvimento da cultura.

No momento da aplicação a cultura se encontrava em início de florescimento, e as plantas daninhas, em pleno desenvolvimento vegetativo, caracterizado pela presença de três a dez folhas e altura inferior a $10 \mathrm{~cm}$, constituindo composição florística em média de 72 , $57,40,28,25$, 3 e 3 plantas por $\mathrm{m}^{2}$, respectivamente, de Commelina benghalensis (trapoeraba), Richardia brasilienses (poaia-branca), Digitaria horizontais (capim-colchão), Brachiaria plantaginea (capim-marmelada), Galinsoga quadriradiata (botão-de-ouro), Ipomoea grandifolia (corda-de-viola) e Emilia sonchifolia (falsa-serralha). Os valores médios de temperatura, umidade relativa do ar e velocidade dos ventos foram, respectivamente, para o início da aplicação sem a proteção do bico de pulverização, de $22,5^{\circ} \mathrm{C}, 88,0 \%$ e $2,5 \mathrm{~km} / \mathrm{h}$ e, no final, de $29,7^{\circ} \mathrm{C}, 68,0 \%$ e $2,8 \mathrm{~km} / \mathrm{h}$. Para aplicação com chapéu-de-napoleão os valores médios de temperatura, umidade relativa do ar e velocidade dos ventos foram, no início, de $29,5{ }^{\circ} \mathrm{C}, 68,0 \%$ e $2,8 \mathrm{~km} / \mathrm{h}$ e, no final, de $31,2{ }^{\circ} \mathrm{C}, 63,0 \%$ e $3,2 \mathrm{~km} / \mathrm{h}$.

As características agronômicas avaliadas no trabalho foram: fitointoxicação (\%) da mamoneira e controle das plantas daninhas aos 10, 20 e 30 dias após a aplicação (DAA), através de escala de notas, em que $0 \%$ significou nenhum efeito de dano às plantas e $100 \%$, morte total, conforme metodologia da SBCPD (1995) e produtividade em $\mathrm{kg} \mathrm{ha}^{-1}$.

Os dados obtidos foram submetidos à análise de variância pelo teste $\mathrm{F}$ e as suas médias comparadas pelo teste de agrupamento de médias de Scott-Knott (Scott \& Knott, 1974), em nivel de $5 \%$ de probabilidade. 


\section{RESULTADOS E DISCUSSÃO}

Na Tabela 2 encontram-se os dados de fitointoxicação da mamoneira cv. Lyra aos 10, 20 e 30 DAA, para as condições de aplicação com e sem chapéu-de-napoleão. Para aplicação sem a proteção, observa-se que os tratamentos com glyphosate $\left(0,72 \mathrm{~kg} \mathrm{ha}^{-1}\right)$, glyphosate $+2,4-$ $\mathrm{D}\left(0,72+0,35 \mathrm{~kg} \mathrm{ha}^{-1}\right)$, glyphosate + flumioxazin $\left(0,72+0,025 \mathrm{~kg} \mathrm{ha}^{-1}\right)$, glyphosate + carfentrazone-ethyl $\left(0,72+0,016 \mathrm{~kg} \mathrm{ha}^{-1}\right)$, glyphosate + diuron $\left(0,72+0,75 \mathrm{~kg} \mathrm{ha}^{-1}\right) \mathrm{e}$ paraquat + diquat $\left(0,20+0,20 \mathrm{~kg} \mathrm{ha}^{-1}\right)$ foram os mais prejudiciais à mamoneira cv. Lyra, causando injúrias visuais superiores a $73,0 \%$ na parte aérea das plantas, a partir dos 10 DAA. Esses efeitos fitotóxicos foram significativamente superiores à condição de aplicação utilizando-se chapéu-de-napoleão, com exceção do tratamento glyphosate + diuron, em que não foram constatadas diferenças significativas aos 10, 20 e 30 DAA entre a forma de aplicação em jato dirigido protegido e sem proteção. A partir dos 10 DAA, também foi possível constatar menores efeitos fitotóxicos para a mistura MSMA + diuron, caracterizando uma classe intermediária pelo teste de Scott-Knott, a qual persistiu até os 30 DAA com danos em torno de $74 \%$, não havendo distinção em relação às formas de aplicação. As misturas paraquat + bentazon e paraquat + diuron apresentaram os melhores resultados de seletividade para mamoneira cv. Lyra em relação aos demais tratamentos, com destaque principalmente para a mistura paraquat + bentazon, independentemente do uso ou não da proteção na aplicação em jato dirigido. Quanto às misturas paraquat + diuron e glyphosate $+2,4-D$, foi significativo o aumento da seletividade constatada, através de aspectos visuais, para aplicação em jato dirigido com auxílio de chapéude-napoleão, em relação à aplicação sem proteção, apesar de os niveis de danos ainda terem se mantido elevados até os 30 DAA.

Os efeitos fitotóxicos sobre a mamoneira cv. Lyra, assim como os desempenhos de controle de plantas daninhas das diferentes misturas de herbicidas estudadas em aplicação em jato dirigido com e sem proteção do bico de pulverização aos 20 DAA, podem ser ilustrados através da Figura 1. Para os tratamentos que envolveram o herbicida glyphosate isolado e/ ou em mistura, os sintomas de danos nas plantas de mamoneira se caracterizaram pela morte do ápice e racemos de algumas plantas, seguido de clorose entre nervuras e necrose dos bordos das folhas mais novas de várias plantas, sobretudo para menor porte e ausência do uso da proteção do bico de pulverização. Nos tratamentos com MSMA + diuron, paraquat + bentazon, paraquat + diuron e paraquat + diquat, os efeitos fitotóxicos foram mais intensos apenas nas folhas mais velhas, devido ao maior contato da pulverização com as folhas inferiores, havendo elevado nivel de desfolha das plantas menores. O tratamento paraquat + bentazon se destacou pelo menor nivel de dano à parte aérea da mamoneira, em relação aos demais tratamentos e condições de aplicação. Esta mistura em tanque apresentou como sintoma característico apenas a coloração enegrecida da base do caule das plantas, onde casualmente houve contato direto da aplicação, independentemente do uso ou não da proteção do bico de pulverização com chapéu-denapoleão.

No que se refere ao controle das plantas daninhas aos 10, 20 e 30 DAA (Tabela 3), todas as misturas de herbicidas de ação total aplicadas com auxilio da proteção do chapéu-de-napoleão apresentaram aos 10 DAA controle eficiente (> 90,0\%) da infestação total, sendo significativamente superior ao do glyphosate isolado, que obteve apenas controle satisfatório $(\leq 80,0 \%)$. Aos 20 DAA, todas as misturas obtiveram controle excelente (> 96,7\%) para aplicação com e sem proteção do bico de pulverização, superando a ação apenas satisfatória do glyphosate $\left(0,72 \mathrm{~kg} \mathrm{ha}^{-1}\right)$ em aplicação isolada, que também obteve controle eficiente na ausência da proteção. Aos 30 DAA, todas as misturas continuaram a promover controle eficiente da infestação para aplicação com e sem chapéu-de-napoleão, superando ainda o glyphosate isolado, principalmente a presença da espécie trapoeraba na infestação. Em função de surgimento de rebrotes, principalmente de trapoeraba e corda-de-viola, as misturas paraquat + bentazon, paraquat + diquat e MSMA + diuron igualaram-se significativamente ao glyphosate isolado na condição com uso de chapéu-de-napoleão, formando um agrupamento pelos critérios de Scott-Knott. 
Tabela 1 - Nomes comum e comercial das misturas em tanque dos herbicidas pulverizados com e sem uso de chapéu-de-napoleão, em aplicação de jato dirigido para controle de plantas daninhas na cultura da mamoneira. Garça-SP, 2006

\begin{tabular}{|c|c|c|c|}
\hline Nome químico & Nome comercial & $\begin{array}{c}\text { Dose } \\
\left(\mathrm{kg} \text { e.a. ou i.a ha }{ }^{-1}\right)\end{array}$ & $\begin{array}{c}\text { Dose } \\
\left(\mathrm{L} \mathrm{ou} \mathrm{kg} \mathrm{pc} \mathrm{h}^{-1}\right)\end{array}$ \\
\hline Glyphosate & Roundup Original $^{\mathbb{B}}$ & 0,72 & 2,0 \\
\hline Glyphosate + 2,4-D & Roundup Original $^{\mathbb{R}}+$ DMA $806^{(\mathbb{R}}$ & $0,72+0,350$ & $2,0+0,50$ \\
\hline Glyphosate + flumioxazin & Roundup Original $^{\mathbb{B}}+$ Flumizyn $^{\circledR}$ & $0,72+0,025$ & $2,0+0,05$ \\
\hline Glyphosate + carfentrazone-ethyl & Roundup Original $^{\circledR}+$ Aurora $400 \mathrm{CE}^{\circledR}$ & $0,72+0,016$ & $2,0+0,04$ \\
\hline Glyphosate + diuron & Roundup Original $^{(\mathbb{B}}+$ Diuron Nortox $500 \mathrm{SC}^{(\mathbb{B})}$ & $0,72+0,750$ & $2,0+1,50$ \\
\hline MSMA + diuron & Dessecan $^{\circledR}+$ Diuron Nortox $500 \mathrm{SC}^{\circledR}$ & $1,44+0,750$ & $3,0+1,50$ \\
\hline Paraquat + diuron & Gramocil $^{(B)}$ & 0,90 & 3,0 \\
\hline Paraquat + diquat & Gramoxone $^{\circledR}+$ Reglone $^{\circledR}$ & $0,20+0,20$ & $1,0+1,00$ \\
\hline Paraquat + bentazon & Gramoxone $^{\circledR}+$ Basagran $600^{\circledR}$ & $0,40+0,48$ & $2,0+0,80$ \\
\hline Testemunha capinada & - & - & - \\
\hline
\end{tabular}

Nos tratamentos a base de glyphosate foi utilizada na calda de pulverização $0,50 \% \mathrm{v} / \mathrm{v}$ de óleo mineral Assist ${ }^{\circledR}$, e nos tratamentos à base de MSMA e Paraquat, o espalhante adesivo $\mathrm{Agral}^{\circledR}$, na dose de $0,25 \% \mathrm{v} / \mathrm{v}$.

Tabela 2 - Porcentagem de fitointoxicação da mamoneira cv. Lyra aos 10, 20 e 30 DAA, submetida ao contraste entre a utilização de aplicação em jato dirigido com e sem chapéu-de-napoleão e misturas de herbicidas de ação total. Garça-SP, 2006

\begin{tabular}{|c|c|c|c|c|c|c|}
\hline \multirow[b]{3}{*}{ Tratamento } & \multicolumn{6}{|c|}{ Fitointoxicação (\%) } \\
\hline & \multicolumn{2}{|c|}{$10 \mathrm{DAA}$} & \multicolumn{2}{|c|}{$20 \mathrm{DAA}$} & \multicolumn{2}{|c|}{$30 \mathrm{DAA}$} \\
\hline & $\begin{array}{c}\text { COM } \\
\text { chapéu-de- } \\
\text { napoleão }\end{array}$ & $\begin{array}{c}\text { SEM } \\
\text { chapéu-de- } \\
\text { napoleão }\end{array}$ & $\begin{array}{c}\text { COM } \\
\text { chapéu-de- } \\
\text { napoleão }\end{array}$ & $\begin{array}{c}\text { SEM } \\
\text { chapéu-de- } \\
\text { napoleão }\end{array}$ & $\begin{array}{c}\text { COM } \\
\text { chapéu-de- } \\
\text { napoleão }\end{array}$ & $\begin{array}{c}\text { SEM } \\
\text { chapéu-de- } \\
\text { napoleão }\end{array}$ \\
\hline Glyphosate & $49,2 \mathrm{~B} \mathrm{~b}$ & $81,2 \mathrm{~A} \mathrm{a}$ & $75,0 \mathrm{~A} \mathrm{a}$ & $88,2 \mathrm{~A} \mathrm{a}$ & $68,7 \mathrm{~A} \mathrm{a}$ & $83,2 \mathrm{~A} \mathrm{~b}$ \\
\hline Glyphosate + 2,4-D & $47,0 \mathrm{~B} \mathrm{~b}$ & $73,0 \mathrm{~A} \mathrm{a}$ & $55,0 \mathrm{~B} \mathrm{~b}$ & $81,2 \mathrm{~A} \mathrm{~b}$ & $51,2 \mathrm{~B} \mathrm{~b}$ & $76,2 \mathrm{~A} \mathrm{~b}$ \\
\hline Glyphosate + flumioxazin & $66,2 \mathrm{~B} \mathrm{a}$ & $85,0 \mathrm{~A} \mathrm{a}$ & $78,7 \mathrm{~A} \mathrm{a}$ & $93,7 \mathrm{~A} \mathrm{a}$ & $81,2 \mathrm{~A} \mathrm{a}$ & $90,5 \mathrm{~A} \mathrm{a}$ \\
\hline Glyphosate + carfentrazone & $59,5 \mathrm{~B} \mathrm{~b}$ & $85,2 \mathrm{~A} \mathrm{a}$ & $61,2 \mathrm{~B} \mathrm{~b}$ & $85,2 \mathrm{~A} \mathrm{a}$ & $76,2 \mathrm{~A} \mathrm{a}$ & $89,5 \mathrm{~A} \mathrm{a}$ \\
\hline Glyphosate + diuron & $78,7 \mathrm{~A} \mathrm{a}$ & $87,7 \mathrm{~A} \mathrm{a}$ & $93,5 \mathrm{~A} \mathrm{a}$ & $97,7 \mathrm{~A} \mathrm{a}$ & $92,7 \mathrm{~A} \mathrm{a}$ & $95,5 \mathrm{~A} \mathrm{a}$ \\
\hline MSMA + diuron & $48,7 \mathrm{~A} \mathrm{~b}$ & $37,2 \mathrm{~A} \mathrm{~b}$ & $65,5 \mathrm{~A} \mathrm{~b}$ & $68,7 \mathrm{~A} \mathrm{~b}$ & $73,7 \mathrm{~A} \mathrm{a}$ & $75,0 \mathrm{~A} \mathrm{~b}$ \\
\hline Paraquat + diuron & $26,2 \mathrm{~B} \mathrm{c}$ & $51,2 \mathrm{~A} \mathrm{~b}$ & $43,2 \mathrm{~B} \mathrm{~b}$ & $76,5 \mathrm{~A} \mathrm{~b}$ & $40,0 \mathrm{~B} \mathrm{c}$ & $72,0 \mathrm{~A} \mathrm{~b}$ \\
\hline Paraquat + diquat & $33,7 \mathrm{~B} \mathrm{c}$ & $83,0 \mathrm{~A} \mathrm{a}$ & $28,7 \mathrm{~B} \mathrm{c}$ & $95,0 \mathrm{~A} \mathrm{a}$ & $25,0 \mathrm{~B} \mathrm{c}$ & $95,7 \mathrm{~A} \mathrm{a}$ \\
\hline Paraquat + bentazon & $10,0 \mathrm{~A} \mathrm{~d}$ & $17,0 \mathrm{~A} \mathrm{c}$ & $4,5 \mathrm{~A} \mathrm{~d}$ & $10,7 \mathrm{~A} \mathrm{c}$ & $3,0 \mathrm{~A} \mathrm{~d}$ & $9,0 \mathrm{~A} \mathrm{c}$ \\
\hline Testemunha capinada & $0,0 \mathrm{~A} \mathrm{~d}$ & $0,0 \mathrm{~A} \mathrm{c}$ & $0,0 \mathrm{~A} \mathrm{~d}$ & $0,0 \mathrm{~A} \mathrm{c}$ & $0,0 \mathrm{~A} \mathrm{~d}$ & $0,0 \mathrm{~A} \mathrm{c}$ \\
\hline Herbicidas $(\mathrm{H})$ & \multicolumn{2}{|c|}{$46,2 *$} & \multicolumn{2}{|c|}{$52,8^{*}$} & \multicolumn{2}{|c|}{$65,6^{*}$} \\
\hline Tecnol. aplicação (TA) & \multicolumn{2}{|c|}{$52,3 *$} & \multicolumn{2}{|c|}{$48,2 *$} & \multicolumn{2}{|c|}{$48,1 *$} \\
\hline $\mathrm{H} \times \mathrm{TA}$ & \multicolumn{2}{|c|}{$4,7 *$} & \multicolumn{2}{|c|}{$5,2 *$} & \multicolumn{2}{|c|}{$7,2 \mathrm{NS}$} \\
\hline $\mathrm{CV}(\%)$ & \multicolumn{2}{|c|}{22,2} & \multicolumn{2}{|c|}{20,5} & \multicolumn{2}{|c|}{18,8} \\
\hline
\end{tabular}

$\mathrm{DAA}=$ dias após aplicação.

Médias seguidas de mesma letra, minúscula na coluna e maiúscula na linha, para as diferentes épocas de avaliação, pertencem ao mesmo grupo, de acordo com critério de agrupamento de Scott-Knott a 5\% de probabilidade.

$\mathrm{NS}=$ não-significativo; $*=\mathrm{P}<0,05$.

Em termos de produtividade, na Figura 2 encontram-se ilustrados os dados referentes ao contraste entre a tecnologia de aplicação e as misturas de herbicidas, onde se observa que a utilização da proteção do bico de pulverização favoreceu o aumento significativo de todos os tratamentos químicos estudados. Os tratamentos com paraquat + bentazon $(0,40$ $\left.+0,48 \mathrm{~kg} \mathrm{ha}^{1}\right)$, glyphosate $\left(0,72 \mathrm{~kg} \mathrm{ha}^{-1}\right)$, glyphosate $+2,4-\mathrm{D}\left(0,72+0,35 \mathrm{~kg} \mathrm{ha}^{-1}\right)$ e paraquat 


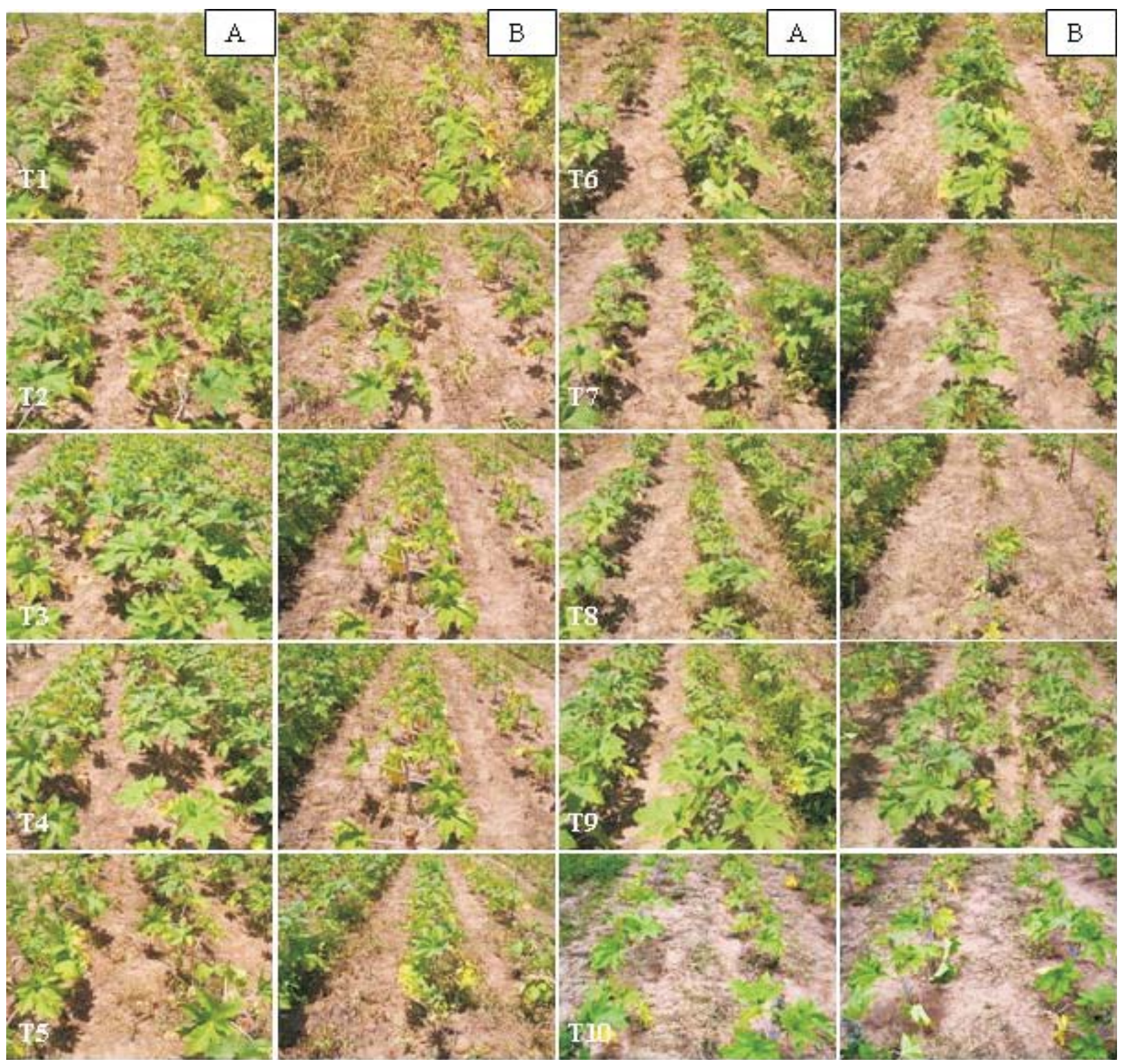

$\mathrm{T} 1=$ glyphosate $\left(0,72 \mathrm{~kg} \mathrm{ha}^{-1}\right) ; \mathrm{T} 2=$ glyphosate $+2,4-\mathrm{D}\left(0,72+0,35 \mathrm{~kg} \mathrm{ha}^{-1}\right) ; \mathrm{T} 3=$ glyphosate + flumioxazin $\left(0,72+0,025 \mathrm{~kg} \mathrm{ha}^{-1}\right) ;$ $\mathrm{T} 4=$ glyphosate + carfentrazone-ethyl $\left(0,72+0,016 \mathrm{~kg} \mathrm{ha}^{-1}\right) ; \mathrm{T} 5=$ glyphosate + diuron $\left(0,72+0,75 \mathrm{~kg} \mathrm{ha}^{1}\right)$; T6 $=$ MSMA + diuron $\left(1,44+0,75 \mathrm{~kg} \mathrm{ha}^{-1}\right) ; \mathrm{T} 7=$ paraquat + diuron $\left(0,9 \mathrm{~kg} \mathrm{ha}^{-1}\right) ; \mathrm{T} 8=$ paraquat + diquat $\left(0,20+0,20 \mathrm{~kg} \mathrm{ha}^{-1}\right) ; \mathrm{T} 9=$ paraquat + bentazon $(0,40$ $\left.0,48 \mathrm{~kg} \mathrm{ha}^{-1}\right) ; \mathrm{T} 10=$ testemunhas capinadas.

Figura 1 - Sintomas de fitointoxicação e controle das plantas daninhas na cultura da mamoneira cv. Lyra aos 20 DAA, submetida ao contraste entre a utilização de aplicação em jato dirigido com (A) e sem (B) chapéu-de-napoleão e misturas de herbicidas de ação total. Garça-SP, 2006.

+ diquat $\left(0,20+0,20 \mathrm{~kg} \mathrm{ha}^{-1}\right)$, aplicados em jato dirigido com bico protegido por chapéu-de-napoleão, proporcionaram os maiores níveis de produtividades da mamoneira cv. Lyra, não diferindo significativamente entre si, quando comparados à testemunha sem aplicação. Entretanto, considerando em conjunto os aspectos de fitointoxicação, controle e produtividade, assim como de menor risco em função do mecanismo de ação dos produtos, as misturas de paraquat + bentazon e paraquat + diquat se destacam entre os tratamentos estudados como as melhores opções. As misturas de glyphosate + flumioxazin $\left(0,72+0,025 \mathrm{~kg} \mathrm{ha}^{-1}\right)$, glyphosate + carfentrazone-ethyl $(0,72+$ $\left.0,016 \mathrm{~kg} \mathrm{ha}^{-1}\right)$, glyphosate + diuron $(0,72+$ 
Tabela 3 - Porcentagem de controle das plantas daninhas aos 10, 20 e 30 DAA, submetida ao contraste entre a utilização de aplicação em jato dirigido com e sem chapéu-de-napoleão e misturas de herbicidas de ação total. Garça-SP, 2006

\begin{tabular}{|c|c|c|c|c|c|c|}
\hline \multirow[b]{3}{*}{ Tratamento } & \multicolumn{6}{|c|}{ Fitointoxicação (\%) } \\
\hline & \multicolumn{2}{|c|}{$10 \mathrm{DAA}$} & \multicolumn{2}{|c|}{$20 \mathrm{DAA}$} & \multicolumn{2}{|c|}{30 DAA } \\
\hline & $\begin{array}{c}\text { COM } \\
\text { chapéu-de- } \\
\text { napoleão }\end{array}$ & $\begin{array}{c}\text { SEM } \\
\text { chapéu-de- } \\
\text { napoleão }\end{array}$ & $\begin{array}{c}\text { COM } \\
\text { chapéu-de- } \\
\text { napoleão }\end{array}$ & $\begin{array}{c}\text { SEM } \\
\text { chapéu-de- } \\
\text { napoleão }\end{array}$ & $\begin{array}{c}\text { COM } \\
\text { chapéu-de- } \\
\text { napoleão }\end{array}$ & $\begin{array}{c}\text { SEM } \\
\text { chapéu-de- } \\
\text { napoleão }\end{array}$ \\
\hline Glyphosate & $80,0 \mathrm{~A} \mathrm{~b}$ & $75,7 \mathrm{~A} \mathrm{~d}$ & $86,7 \mathrm{~A} \mathrm{~b}$ & $81,7 \mathrm{~A} \mathrm{c}$ & $89,5 \mathrm{~A} \mathrm{~b}$ & $79,0 \mathrm{~B} \mathrm{~b}$ \\
\hline Glyphosate + 2,4-D & $97,0 \mathrm{~A} \mathrm{a}$ & $92,5 \mathrm{~A} \mathrm{~b}$ & $96,7 \mathrm{~A} \mathrm{a}$ & $95,0 \mathrm{~A} \mathrm{a}$ & $97,7 \mathrm{~A} \mathrm{a}$ & $96,7 \mathrm{~A} \mathrm{a}$ \\
\hline Glyphosate + flumioxazin & $100,0 \mathrm{~A} \mathrm{a}$ & $99,0 \mathrm{~A} \mathrm{a}$ & $99,5 \mathrm{~A} \mathrm{a}$ & $98,0 \mathrm{~A} \mathrm{a}$ & $97,5 \mathrm{~A} \mathrm{a}$ & $97,0 \mathrm{~A} \mathrm{a}$ \\
\hline Glyphosate + carfentrazone & $98,7 \mathrm{~A} \mathrm{a}$ & $99,2 \mathrm{~A} \mathrm{a}$ & $100,0 \mathrm{~A} \mathrm{a}$ & $100,0 \mathrm{~A} \mathrm{a}$ & $97,0 \mathrm{~A} \mathrm{a}$ & $99,5 \mathrm{~A} \mathrm{a}$ \\
\hline Glyphosate + diuron & $93,7 \mathrm{~A} \mathrm{a}$ & $88,7 \mathrm{~B} \mathrm{c}$ & $97,7 \mathrm{~A} \mathrm{a}$ & $92,5 \mathrm{~B} \mathrm{~b}$ & $97,0 \mathrm{~A} \mathrm{a}$ & $95,7 \mathrm{~A} \mathrm{a}$ \\
\hline MSMA + diuron & $95,0 \mathrm{~A} \mathrm{a}$ & $85,0 \mathrm{~B} \mathrm{c}$ & $99,5 \mathrm{~A} \mathrm{a}$ & $91,7 \mathrm{~B} \mathrm{~b}$ & $94,2 \mathrm{~A} \mathrm{~b}$ & $87,0 \mathrm{~B} \mathrm{~b}$ \\
\hline Paraquat + diuron & $100,0 \mathrm{~A} \mathrm{a}$ & $98,7 \mathrm{~A} \mathrm{a}$ & $99,5 \mathrm{~A} \mathrm{a}$ & $98,2 \mathrm{~A} \mathrm{a}$ & $99,5 \mathrm{~A} \mathrm{a}$ & $96,5 \mathrm{~A} \mathrm{a}$ \\
\hline Paraquat + diquat & $98,7 \mathrm{~A} \mathrm{a}$ & $97,5 \mathrm{~A} \mathrm{a}$ & $98,0 \mathrm{~A} \mathrm{a}$ & $97,0 \mathrm{~A} \mathrm{a}$ & $94,7 \mathrm{~A} \mathrm{~b}$ & $93,2 \mathrm{~A} \mathrm{a}$ \\
\hline Paraquat + bentazon & $96,7 \mathrm{~A} \mathrm{a}$ & $90,0 \mathrm{~B} \mathrm{c}$ & $97,0 \mathrm{~A} \mathrm{a}$ & $88,2 \mathrm{~B} \mathrm{~b}$ & $93,5 \mathrm{~A} \mathrm{~b}$ & $92,2 \mathrm{~A} \mathrm{a}$ \\
\hline Testemunha capinada & $100,0 \mathrm{~A} \mathrm{a}$ & $100,0 \mathrm{~A} \mathrm{a}$ & $100,0 \mathrm{~A} \mathrm{a}$ & $100,0 \mathrm{~A} \mathrm{a}$ & $100,0 \mathrm{~A} \mathrm{a}$ & $100,0 \mathrm{~A} \mathrm{a}$ \\
\hline Herbicidas $(\mathrm{H})$ & \multicolumn{2}{|c|}{$33,2 *$} & \multicolumn{2}{|c|}{$13,8 *$} & \multicolumn{2}{|c|}{$10,1 *$} \\
\hline Tecnol. aplicação (TA) & \multicolumn{2}{|c|}{$20,0 *$} & \multicolumn{2}{|c|}{$16,1 *$} & \multicolumn{2}{|c|}{$6,5 *$} \\
\hline $\mathrm{H} \times \mathrm{TA}$ & \multicolumn{2}{|c|}{$2,0 \mathrm{NS}$} & \multicolumn{2}{|c|}{$1,6 \mathrm{NS}$} & \multicolumn{2}{|c|}{$1,6 \mathrm{NS}$} \\
\hline $\mathrm{CV}(\%)$ & \multicolumn{2}{|c|}{3,5} & \multicolumn{2}{|c|}{3,7} & \multicolumn{2}{|c|}{4,4} \\
\hline
\end{tabular}

DAA $=$ dias após aplicação.

Médias seguidas de mesma letra, minúscula na coluna e maiúscula na linha, para as diferentes épocas de avaliação, pertencem ao mesmo grupo, de acordo com critério de agrupamento de Scott-Knott a $5 \%$ de probabilidade.

$\mathrm{NS}=$ não-significativo; $*=\mathrm{P}<0,05$.

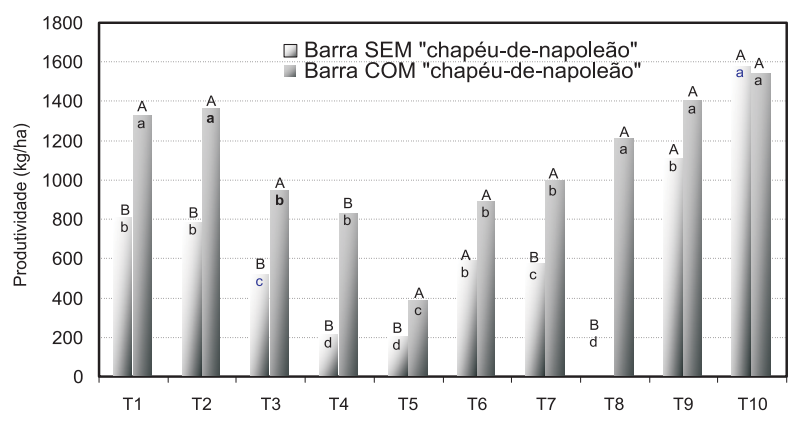

$\mathrm{T} 1=$ glyphosate $\left(0,72 \mathrm{~kg} \mathrm{ha}^{-1}\right) ; \mathrm{T} 2=$ glyphosate $+2,4-\mathrm{D}(0,72+$ $\left.0,35 \mathrm{~kg} \mathrm{ha}^{-1}\right) ; \mathrm{T} 3=$ glyphosate + flumioxazin $\left(0,72+0,025 \mathrm{~kg} \mathrm{ha}^{-1}\right)$; $\mathrm{T} 4=$ glyphosate + carfentrazone-ethyl $\left(0,72+0,016 \mathrm{~kg} \mathrm{ha}^{-1}\right)$ $\mathrm{T} 5=$ glyphosate + diuron $\left(0,72+0,75 \mathrm{~kg} \mathrm{ha}^{1}\right) ;$ T6 $=$ MSMA + diuron $\left(1,44+0,75 \mathrm{~kg} \mathrm{ha}^{-1}\right) ; \mathrm{T} 7=$ paraquat + diuron $\left(0,9 \mathrm{~kg} \mathrm{ha}^{-1}\right)$; $\mathrm{T} 8=$ paraquat + diquat $\left(0,20+0,20 \mathrm{~kg} \mathrm{ha}^{-1}\right) ; \mathrm{T} 9=$ paraquat + bentazon $\left(0,40+0,48 \mathrm{~kg} \mathrm{ha}^{-1}\right)$; T10 $=$ testemunhas capinadas.

Figura 2 - Produtividade ( $\left.\mathrm{kg} \mathrm{ha}^{-1}\right)$ de mamoneira cv. Lyra, submetida ao contraste entre a utilização de aplicação em jato dirigido com e sem chapéu-de-napoleão e misturas de herbicidas de ação total. Garça-SP, 2006. $\left.0,75 \mathrm{~kg} \mathrm{ha}^{-1}\right)$, MSMA + diuron $(1,44+$ $\left.0,75 \mathrm{~kg} \mathrm{ha}^{-1}\right)$ e diuron + paraquat $\left(0,90 \mathrm{~kg} \mathrm{ha}^{1}\right)$ apresentaram as maiores limitações para aplicações de jato dirigido, mesmo quando associadas ao efeito de proteção do bico de pulverização por chapéu-de-napoleão.

Nesse sentido, pode-se constatar que o uso da proteção do bico de pulverização, em aplicações de jato dirigido, pode viabilizar a utilização de alguns produtos de ação total, considerados extremamente prejudiciais para a cultura da mamoneira de porte anão, como glyphosate, glyphosate + 2,4-D e paraquat + diquat. Contudo, é importante ressaltar que essa possibilidade de associação de misturas de produtos de ação total e uso de proteção do bico de pulverização por chapéu-de-napoleão é uma condição de seletividade de posicionamento, a qual será extremamente influenciada por fatores como regularidade do porte da cultura, 
características da infestação e uniformidade da pulverização.

De forma geral, através dos resultados obtidos, pode-se concluir que o herbicida glyphosate e as misturas paraquat + bentazon, glyphosate $+2,4-\mathrm{D}$ e paraquat + diquat $(0,20+$ $0,20 \mathrm{~kg} \mathrm{ha}^{-1}$ ) proporcionaram os maiores níveis de produtividade da mamoneira cv. Lyra quando aplicados em jato dirigido com auxílio da proteção do chapéu-de-napoleão. Entretanto, considerando em conjunto aspectos de fitointoxicação, controle da infestação, produtividade e menor risco em função do mecanismo de ação dos produtos, as misturas paraquat + bentazon $\left(0,40+0,48 \mathrm{~kg} \mathrm{ha}^{-1}\right)$ e paraquat $+\mathrm{di}-$ quat $\left(0,20+0,20 \mathrm{~kg} \mathrm{ha}^{-1}\right)$ foram as mais indicadas para aplicação em jato dirigido com chapéude-napoleão.

\section{LITERATURA CITADA}

AMARAL, J. G. C. Cultivar de mamona AL Guarany 2002. CATI Responde, n. 52, p. 1-2, 2002. Disponível em: <http:/ /www.cati.sp.gov.br/tecnologias/catiresponde/ CR52mamona_Guarany.htm>. Acesso em: 31 mar. 2004.

AZEVEDO, D. M. P. et al. Plantas daninhas e seu controle In: AZEVEDO, D. M. P.; LIMA, E. F. O agronegócio da mamona no Brasil. Brasília: Embrapa Informação Tecnológica, 2001. p. 161-189.

BELTRÃO, N. E. M. et al. Fitologia. In: AZEVEDO, D. M. P.; LIMA, E. L. (Eds.). O agronegócio da mamona no Brasil. Brasília: Embrapa Informação Tecnológica, 2001. p. 37-61.
FREIRE, E. C.; LIMA, E. F.; ANDRADE, F. P.

Melhoramento genético. In: AZEVEDO, D. M. P.; LIMA, E. F. (Orgs.). O agronegócio da mamona no Brasil. Brasília: Embrapa Algodão, 2001. p. 229-256.

GEMINI, G. A. Herbicidas - Indicações básicas. Campinas: CATI, 1985. 251 p. (Documento Técnico, 52).

MACIEL, C. D. G. et al. Seletividade de herbicidas em cultivares de mamona. R. bras. ol. fibros, v. 11, n. 1, p. 4754,2007

MARTINS, F. M. et al. Seletividade de herbicidas graminicidas aplicados em pós-emergência na cultura da mamona. In: CONGRESSO BRASILEIRO DA CIÊNCIA DAS PLANTAS DANINHAS, 24., 2004. São Pedro. Anais... Londrina: Sociedade Brasileira da Ciência das Plantas Daninhas, 2004. CD-ROM.

SAVY FILHO, A. Mamona (Ricinus communis L.). In FAHL, J. I. et al. (Eds.). Instrumentações agrícolas para as principais culturas econômicas. 6.ed. Campinas: Instituto Agronômico, 1998. 396 p. (Boletim, 200).

SOCIEDADE BRASILEIRA DA CIÊNCIA DAS PLANTAS DANINHAS - SBCPD. Procedimentos para instalação, avaliação e análise de experimentos com herbicidas. Londrina: 1995. 42 p.

SCOTT, A.; KNOTT, M. Cluster-analysis method for grouping means in analysis of variance. Biometrics, v. 30, n. 3 , p. $507-512,1974$

WEISS, E. A. Oilseed crops. London: Longman, 1983. $660 \mathrm{p}$.

YAROSLAVSKAYA, P. N. Methods of growing castor. In: MOSHKIN, V. A. Castor. New Delhi: Amerind, 1986 p. $161-184$ 